\title{
Heavy quark masses from lattice QCD
}

\author{
Andrew T. Lytle ${ }^{1, a}$ \\ ${ }^{1}$ SUPA, School of Physics and Astronomy \\ University of Glasgow, Glasgow, G12 8QQ, UK
}

\begin{abstract}
Progress in quark mass determinations from lattice QCD is reviewed, focusing on results for charm and bottom mass. These are of particular interest for precision Higgs studies. Recent determinations have achieved percent-level uncertainties with controlled systematics. Future prospects for these calculations are also discussed.
\end{abstract}

\section{Introduction}

Quark masses are fundamental parameters of QCD, arising in the Standard Model (SM) from Yukawa interactions with the Higgs field. LHC measurements of the Higgs' couplings are consistent with SM predictions, but high-precision studies at future accelerators such as the ILC will measure couplings at the per mil level [1]. In order to test the SM at this level, and discriminate potential new physics effects, correspondingly precise determinations of quark mass are required.

Lattice calculations of quark masses have seen considerable progress in recent years, with charm and bottom uncertainties being quoted at the percent to few-percent level. This is due to a combination of new measurement techniques and increasingly realistic simulations. State-of-the-art simulations include dynamical $u, d, s$, and frequently $c$ quarks, with pion masses at or near their physical values, and typically at several lattice spacings. This realism translates to increasingly accurate results, and with fewer systematic errors. In order to reliably determine quark masses at the sub-percent level, it is important to have multiple calculational techniques/strategies available, as well as independent determinations from different groups.

The outline of the rest of this article is as follows: Sec. 2 briefly discusses quark mass parameters in a general context, and how they are determined in lattice QCD simulations. Sec. 3 discusses recent progress in the charm mass determinations, focusing on a method using current-current correlators. Sec. 4 will look at strategies and results for bottom mass determinations, and Sec. 5 discusses the important role played by mass ratios. Sec. 6 presents a summary and discusses future prospects for these calculations.

\section{Quark mass and LQCD}

Quark masses are scheme and scale dependent quantities that, along with $\alpha_{s}$, specify QCD at the Lagrangian level. These parameters must be determined from comparison of theory and experiment.

\footnotetext{
ae-mail: andrew.lytle@glasgow.ac.uk
} 
In the absence of nonperturbative lattice simulations, one must focus on experimentally measurable observables that are 1) sensitive to quark masses and 2) can be reliably computed in perturbation theory. One set of observables satisfying these criteria are derived from the the R-ratio, for which the relevant perturbation series are known to $\mathrm{N}^{3} \mathrm{LO}$ [2-4]. To be discussed in Sec. 3.1, an effective way to calculate $m_{q}$ for heavy quarks via lattice simulations uses these same perturbative calculations, but substitutes experimental data with data from LQCD simulations.

Lattice QCD simulations are well suited for mass determinations, since the mass parameters are simulation inputs controlled by the "experimenter". By changing the input masses, one can measure the resultant change in physical observables such as meson masses. In a standard LQCD simulation, one tunes the input masses in order to reproduce a number of low energy observables - one for each quark in the theory. In this way one obtains (typically very precise) bare quark masses, but in the particular lattice regularization one happens to be using. In order to make contact with a continuumregularized determination such as the $\overline{\mathrm{MS}}$ scheme, one needs an additional calculation of the lattice to $\overline{\mathrm{MS}}$ matching factor. This can be found using lattice perturbation theory or via non-perturbative renormalization (NPR) techniques. The ratios of bare quark masses in a given regularization are however immediately useful, as they are, up to lattice artfifacts, equal to renormalized mass ratios.
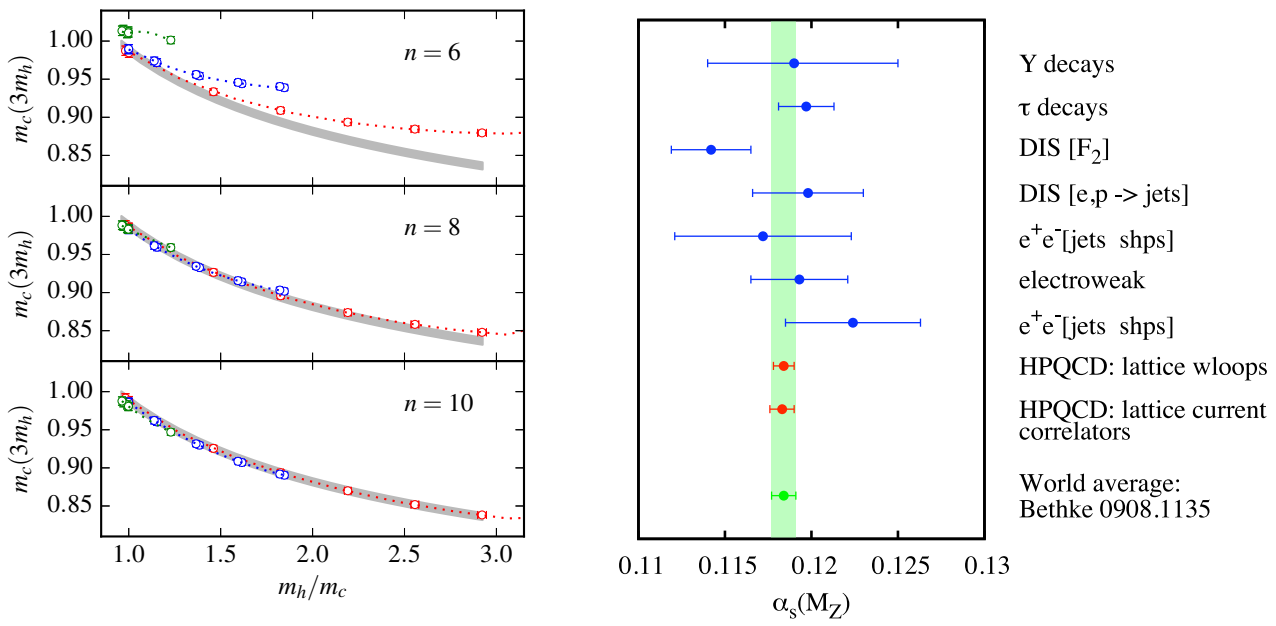

Figure 1. (Left) Data from [5] showing $m_{c}^{\overline{\mathrm{MS}}}\left(\mu=3 m_{h}\right)$ extracted from lattice data and perturbation theory for moments $n=6,8,10$ using Eq. (8). The green/blue/red data points correspond to lattice spacings of 0.12/0.09/0.06 $\mathrm{fm}$. The gray band shows the evolution of the best-fit value for $m_{c}$ using perturbation theory. (Right) Value of $\alpha_{s}^{\overline{\mathrm{MS}}}\left(M_{Z}\right)$ from [5] compared with determinations based on various experimental inputs and a world average.

\section{Charm quark mass}

\subsection{Current-current correlator method.}

The current-current correlator method uses time moments of Euclidean-time twopoint functions,

$$
G(t)=a^{6} \sum_{\mathbf{x}}\left(a m_{0 h}\right)^{2}\left\langle J_{5}(t, \mathbf{x}) J_{5}(0,0)\right\rangle
$$


Here $J_{5} \equiv \bar{\psi}_{h} \gamma_{5} \psi_{h}, a$ is the lattice spacing, and $a m_{0 h}$ is the bare quark mass parameter in lattice units. In formalisms with sufficient chiral symmetry, the current $J_{5}$ is absolutely normalized. The correlator $G(t)$ is UV finite, so that

$$
G(t)_{\mathrm{cont}}=G(t)_{\mathrm{latt}}+O\left(a^{2}\right) \quad(t \neq 0) .
$$

The correlators $G(t)_{\text {latt }}$ are the same ones used in the computation of pseudoscalar masses and decay constants, in which case it is the large- $t$ exponential tail of the correlator that is of interest. For the mass calculation it is the small- $t$ short distance behavior that is extracted via time-moments of $G(t)$, defined as:

$$
G_{n, \text { latt }}=\sum_{t=0}^{T}(t / a)^{n} G(t)_{\text {latt }} .
$$

The time-moments $G_{n}$ have also been computed to $\mathrm{N}^{3} \mathrm{LO}$ in perturbation theory [2-4]. For $n \geq 4$,

$$
G_{n, \text { pert }}=\frac{g_{n}\left(\alpha_{\overline{\mathrm{MS}}}, \mu\right)}{\left(\operatorname{am}_{h}(\mu)\right)^{n-4}} .
$$

Here $g_{n}$ is a series in $\alpha_{s}$ and $m_{h}(\mu)$ is the $\overline{\mathrm{MS}}$ quark mass at the scale $\mu$. The basic strategy to extract the quark mass is to compare $G_{n, \text { cont }}$, the continuum extrapolated $G_{n, \text { latt }}$ values, with the perturbative expressions $G_{n \text {,pert }}$ in Eq. (4) (evaluated at a scale $\mu \sim m_{h}$ ), and from these determine best-fit values for $\alpha_{\overline{\mathrm{MS}}}(\mu)$ and $m_{h}(\mu)$. For example, computing the continuum limit of $G_{4 \text {,latt }}$ with physically tuned input charm masses $m_{0 c}$, one can obtain $\alpha_{\overline{\mathrm{MS}}}\left(m_{c}\right)$, and then use this value in $G_{6}$ to obtain $m_{c}\left(m_{c}\right)$.

The HPQCD collaboration carried out an analysis in [5] using reduced moments, $R_{n}$, which are related simply to the time-moments as

$$
\begin{aligned}
& R_{4}=G_{4} / G_{4}^{(0)} \\
& R_{n}=\frac{1}{m_{0 c}}\left(G_{n} / G_{n}^{(0)}\right)^{1 /(n-4)} \quad(n \geq 6),
\end{aligned}
$$

where $G_{n}^{(0)}$ are the tree-level results for the moments. Dividing by $G_{n}^{(0)}$ has the advantage of reducing lattice-spacing effects. In continuum perturbation theory,

$$
\begin{aligned}
& R_{4}=r_{4}\left(\alpha_{\overline{\mathrm{MS}}}, \mu\right) \\
& R_{n}=\frac{1}{m_{c}(\mu)} r_{n}\left(\alpha_{\overline{\mathrm{MS}}}, \mu\right) \quad(n \geq 6) .
\end{aligned}
$$

Here $r_{n}$ are the perturbative expressions obtained from powers of $g_{n} / g_{n}^{(0)}$, with $g_{n}^{(0)}$ the lowest order perturbative result. For a given $m_{0 h}$ one computes the values $R_{n}$ from Eq. (6) to get estimates of $m_{c}\left(3 m_{h}\right)=R_{n} / r_{n}\left(3 m_{h}\right)$, via Eq. (8) (here the scale $\mu$ is taken to be $\left.3 m_{h}\right)$. 
The running of $m_{c}(\mu)$ was calculated this way in [5] using $n_{f}=2+1+1$ HISQ ensembles. The $n=4,6,8,10$ moments were obtained using three different lattice spacings $a \approx 0.12,0.09,0.06 \mathrm{fm}$ and for seven input masses from $m_{h}=m_{c}$ to $0.7 m_{b}$. The extractions of $m_{c}\left(3 m_{h}\right)$ from each of these data points are shown in Fig. 1 (left), along with the perturbative running. Fig. 1 (right) shows the corresponding estimate of $\alpha_{s}\left(M_{Z}\right)$ extracted from this data, compared with results based on other experimental inputs.

Estimates of $m_{c}(\mu)$ from time-moments are subject to a number of systematic uncertainties. The truncation of perturbation theory limits the precision, but fortunately the expansions of $r_{n}=1+$ $\sum_{j} \alpha^{j} r_{n j}$ are known for $j=1,2,3$ and $n \leq 10$. The lattice moments are sensitive to condensate terms not captured in the perturbative expansions. These effects are suppressed like $\left(\Lambda_{\mathrm{QCD}} / 2 m_{h}\right)^{4}$, but grow with $n$. The lattice data also has cut-off effects, which grow like $\alpha_{s}\left(a m_{h}\right)^{2}$ and decrease with increasing $n$; these trends are visible in Fig. 1.

Fitting the moments data for $n=4,6,8,10$ to Eqs. (7) and (8), HPQCD find

$$
\begin{aligned}
& m_{c}^{\overline{\mathrm{MS}}}\left(3 \mathrm{GeV}, n_{f}=4\right)=0.9851(63) \mathrm{GeV} \\
& \alpha_{s}^{\overline{\mathrm{MS}}}\left(3 \mathrm{GeV}, n_{f}=4\right)=0.2545(37) .
\end{aligned}
$$

These are compatible with earlier $n_{f}=2+1$ results [6]. The compatibility of $n_{f}=2+1$ and $n_{f}=2+1+1$ results suggests that the effect of charm quarks in the sea can be treated perturbatively to this level of precision.

The JLQCD collaboration has also utilized the current-current correlator method with $n_{f}=2+1$ domain-wall fermions to determine $m_{c}$ and $\alpha_{s}$ [7]. Their calculation uses three lattices spacings $a \approx$ $0.08,0.055,0.044 \mathrm{fm}$, and focuses on $R_{6}, R_{8}$, and $R_{10}$, from which they find

$$
\begin{aligned}
& m_{c}^{\overline{\mathrm{MS}}}\left(3 \mathrm{GeV}, n_{f}=3\right)=0.9948(71) \mathrm{GeV} \\
& \alpha_{s}^{\overline{\mathrm{MS}}}\left(3 \mathrm{GeV}, n_{f}=3\right)=0.2514(95) .
\end{aligned}
$$

\subsection{Results comparison}

In [8] the ETMC collaboration used lattice RI/MOM techniques to determine a mass renormalization factor $Z_{m}^{\mathrm{RI}}(\mu, 1 / a)$ connecting the bare mass to the RI-scheme mass, $m_{c}^{\mathrm{RI}}(\mu)=Z_{m}^{\mathrm{RI}}(\mu, 1 / a) m_{c 0}$, which is then converted to the $\overline{\mathrm{MS}}$ scheme using continuum perturbation theory. Unlike the current-current correlator method, which uses a heavy input mass to set the scale $\mu$, the RI/MOM calculation is extrapolated to the chiral limit, and ETMC have generated mass degenerate $n_{f}=4$ ensembles for this purpose. The $\chi \mathrm{QCD}$ collaboration have also used RI/MOM methods for their $n_{f}=2+1$ determination $[9,10]$.

A comparison of recent lattice results for $m_{c}^{\overline{\mathrm{MS}}}$ is shown in Fig. 2.

\section{Bottom mass}

It is challenging to directly simulate the $b$ quark in relativistic lattice simulations, since one would like $a m_{b 0} \ll 1$ in order for discretization effects to be under control. As an alternative, effective theories may be employed such as non-relativistic QCD (NRQCD) or heavy-quark effective theory (HQET). It has also recently become possible to approach the $b$ mass using improved relativistic actions, making extrapolation methods viable. 


\subsection{Ratio method}

The ETMC collaboration have used the ratio method [11] to extrapolate relativistic $n_{f}=2+1+1$ simulation results around the charm mass to the bottom mass [12]. The method is based on the expectation from HQET that

$$
\lim _{m_{h}^{\text {pole }} \rightarrow \infty} \frac{M_{h l}}{m_{h}^{\text {pole }}}=\text { constant },
$$

where $M_{h l}$ is the mass of a heavy-light meson and $m_{h}^{\text {pole }}$ is the heavy quark pole mass.

They calculate ratios of meson masses, $M_{h l}\left(m_{h}\right) / M_{h l}\left(m_{h} / \lambda\right)$, computed for a series of masses $m_{h}$ around the charm mass, e.g.: $m_{h}^{(0)}=m_{c}, m_{h}^{(1)}=\lambda m_{c}, \ldots, m_{h}^{(n)}=\lambda^{n} m_{c}$. These ratios have the advantage that discretization effects proportional to $\left(a m_{h}\right)^{2}$ are largely canceled. From these ratios they construct the function

$$
y\left(m_{h}, \lambda\right)=\lambda^{-1} \frac{M_{h l}\left(m_{h}\right)}{M_{h l}\left(m_{h} / \lambda\right)} \frac{\rho\left(m_{h} / \lambda\right)}{\rho\left(m_{h}\right)} .
$$

The functions $\rho\left(m_{h}\right)$ on the r.h.s. of Eq. (14) relate the pole mass to the $\overline{\mathrm{MS}}$ mass and are known to $\mathrm{N}^{3} \mathrm{LO}$ in perturbation theory. $y\left(m_{h}, \lambda\right)$ satisfies $\lim _{m_{h} \rightarrow \infty} y\left(m_{h}, \lambda\right)=1$ on account of Eq. (13), and so its value can interpolated between the charm region and the static limit using a fit ansatz. Rewriting Eq. (14), the combination $\lambda y\left(m_{h}, \lambda\right) \frac{\rho\left(m_{h}\right)}{\rho\left(m_{h} / \lambda\right)}$ is a known function that evolves $M_{h l}\left(m_{h} / \lambda\right)$ to $M_{h l}\left(m_{h}\right)$. Choosing $\lambda$ such that $M_{h l}\left(m_{h}^{(N)}\right)=M_{b l}^{\text {phys }}$ for some $N$, they determine the $b$ mass from $m_{b}=\lambda^{N} m_{c}$.

\subsection{NRQCD approach}

The NRQCD Hamiltonian is written as an expansion in $v^{2}$, where $v$ is a typical velocity of a $b$ quark in the system of interest. NRQCD calculations should be carried out with $a m_{b 0}>1$, which has the advantage that the $b$ can be simulated using relatively coarse lattices. On the other hand it not as straightforward to extract continuum physics $(a \rightarrow 0$ limit) as compared to relativistic calculations.

The NRQCD current-correlator approach [13] is similar to the relativistic approach described in Sec. 3.1. Here however the currents need to be normalized,

$$
J_{\mu}^{\mathrm{NRQCD}}=Z_{V} J_{\mu}^{\mathrm{cont}} .
$$

Then the time moments are related to continuum perturbation theory,

$$
G_{n}^{\mathrm{NRQCD}}=Z_{\mathrm{V}}^{2} \frac{g_{n}(\alpha \overline{\mathrm{MS}}, \mu)}{\left(a m_{b}(\mu)\right)^{n-2}} .
$$

The factors of $Z_{V}$ can be canceled by constructing ratios of successive moments. Because the continuum limit cannot be approached directly one instead studies $m_{b}$ as a function of the moment number. Compared to the charm case, condensate contributions which grow with moment number are more suppressed at the heavier quark mass. A "plateau" in $m_{b}$ as a function of moment number implies that $n$ is sufficiently large for discretization effects to be small. Such a plateau from [13] is shown in Fig. 3 (left).

Results at three lattice spacings and with two different light-quark masses for $n=18$ are shown in Fig. 3 (right). A fit to this data, including systematic errors, and perturbatively evolved to $m_{b}$ gives

$$
m_{b}^{\overline{\mathrm{MS}}}\left(m_{b}^{\overline{\mathrm{MS}}}, n_{f}=5\right)=4.196(23) \mathrm{GeV} .
$$

This result is compared with others in Fig. 4. It is significant that the values in the figure are calculated using a range of techniques. In [6] results are extrapolated to $m_{b}$ from below, using a 

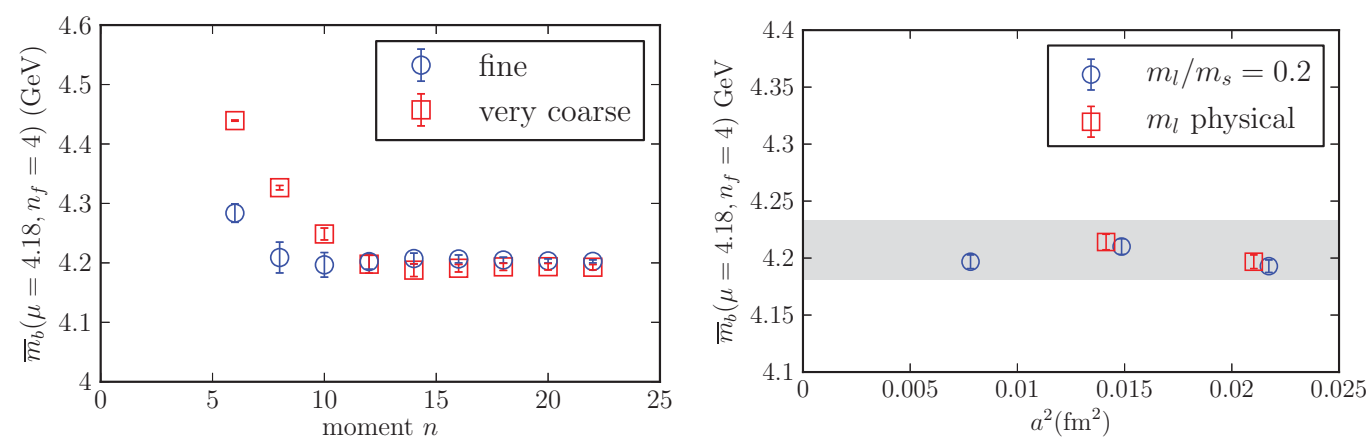

Figure 3. (Left) $m_{b}^{\overline{\mathrm{MS}}}$ extracted from the moments of NRQCD current-current correlators at two different lattice spacings from [13]. (Right) Results from the $n=18$ moment as a function of lattice spacing and for two different light-quark masses. The gray band gives the continuum determination with the total error budget.

relativistic action as described in Sec. 3.1. This calculation is based on a different range of moment numbers, and uses a different action than [13]. The work of [14] uses the binding energy of $\Upsilon$ and $B_{s}$ mesons, computed using NRQCD and lattice perturbation theory, to determine the heavy quark pole mass, which is then converted to the $\overline{\mathrm{MS}}$ mass with continuum perturbation theory.

\section{Mass ratios}

The bare mass parameters input to lattice QCD simulations are pure numbers $\left(a m_{0}\right)$ corresponding to masses expressed in units of the inverse lattice spacing. These are tuned by requiring that a number of observables, one for each input mass, achieve their physical values, resulting in a set of numbers $\left\{\left(a m_{u d 0}\right),\left(a m_{s 0}\right),\left(a m_{c 0}\right)\right\}$. The bare lattice inputs are defined at the cutoff scale and depend on the details of the discretization. However, ratios of input masses are equal to the ratios of $\overline{\mathrm{MS}}$ masses, up to discretization effects that vanish in the continuum,

$$
\frac{a m 1_{0}}{a m 2_{0}}=\frac{m 1^{\overline{\mathrm{MS}}}(\mu)}{m 2^{\overline{\mathrm{MS}}}(\mu)}+O\left(a^{2}\right) .
$$

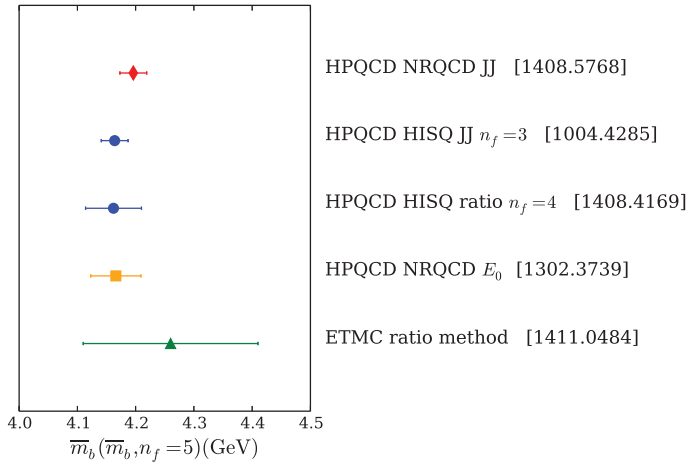

Figure 4. Comparison plot for determinations of $m_{b}^{\overline{\mathrm{MS}}}\left(m_{b}, n_{f}=5\right)$, from $n_{f}=2+1$ and $n_{f}=2+1+1$ simulations.

Once the $\overline{\mathrm{MS}}$ mass is known for one quark in the theory, this can be converted to the $\overline{\mathrm{MS}}$ masses for the others using ratios of the input mass parameters.

In [5] HPQCD use this technique to obtain values for $m_{s}$ and $m_{b}$ using bare mass ratios and a precise determination of $m_{c}$. Taking the continuum limit of $m_{c 0} / m_{s 0}$ they find

$$
\frac{m_{c}\left(\mu, n_{f}\right)}{m_{s}\left(\mu, n_{f}\right)}=11.652(65)
$$


Using their result for $m_{c}^{\overline{\mathrm{MS}}}(\mu)$ from the current-current correlator method discussed in Sec. 3.1, they obtain

$$
m_{s}^{\overline{\mathrm{MS}}}\left(3 \mathrm{GeV}, n_{f}=3\right)=84.7(7) \mathrm{MeV},
$$

which is the most precise estimate to date. Using input mass ratios involving a heavy mass $m_{h 0} / m_{c 0}$, and extrapolating to $m_{b 0}$, they obtain

$$
\begin{aligned}
\frac{m_{b}\left(\mu, n_{f}\right)}{m_{c}\left(\mu, n_{f}\right)} & =4.528(54) \\
m_{b}^{\overline{\mathrm{MS}}}\left(m_{b}, n_{f}=5\right) & =4.162(48) \mathrm{GeV}
\end{aligned}
$$

\section{Conclusion}

Progress in lattice calculations of charm and bottom quark mass was reviewed. In order to achieve reliable (sub-)percent level uncertainties for these quantities, it is important that a variety of calculational strategies are used, and also that independent measurements are carried out by different groups.

At present the most precise quoted values for $c$ mass come from the current-current correlator technique. Precision in the value of the charm mass can be cascaded to the other masses using bare quark-mass ratios, which are determined in the tuning of simulation parameters to the physical point. Calculations of the $b$ mass either use an effective-theory framework for the $b$ quark or extrapolate relativistic simulation results from the lower-mass region where discretization effects are under control. Extrapolation methods will continue to improve as ensembles with smaller lattice spacings become available.

\section{References}

[1] G. P. Lepage, P. B. Mackenzie and M. E. Peskin, arXiv:1404.0319 [hep-ph].

[2] K. G. Chetyrkin, J. H. Kuhn and C. Sturm, Eur. Phys. J. C 48 (2006) 107 [hep-ph/0604234].

[3] R. Boughezal, M. Czakon and T. Schutzmeier, Phys. Rev. D 74 (2006) 074006 [hep$\mathrm{ph} / 0605023]$.

[4] A. Maier, P. Maierhofer, P. Marquard and A. V. Smirnov, Nucl. Phys. B 824 (2010) 1 [arXiv:0907.2117 [hep-ph]].

[5] B. Chakraborty et al., Phys. Rev. D 91 (2015) 5, 054508 [arXiv:1408.4169 [hep-lat]].

[6] C. McNeile, C. T. H. Davies, E. Follana, K. Hornbostel and G. P. Lepage, Phys. Rev. D 82 (2010) 034512 [arXiv:1004.4285 [hep-lat]].

[7] K. Nakayama, B. Fahy and S. Hashimoto, arXiv:1511.09163 [hep-lat].

[8] N. Carrasco et al. [European Twisted Mass Collaboration], Nucl. Phys. B 887 (2014) 19 [arXiv:1403.4504 [hep-lat]].

[9] Y. B. Yang et al., Phys. Rev. D 92 (2015) 3, 034517 [arXiv:1410.3343 [hep-lat]].

[10] Z. Liu et al. [chiQCD Collaboration], Phys. Rev. D 90 (2014) 3, 034505 [arXiv:1312.7628 [heplat]].

[11] B. Blossier et al. [ETM Collaboration], JHEP 1004 (2010) 049 [arXiv:0909.3187 [hep-lat]].

[12] A. Bussone et al., arXiv:1411.0484 [hep-lat].

[13] B. Colquhoun, R. J. Dowdall, C. T. H. Davies, K. Hornbostel and G. P. Lepage, Phys. Rev. D 91 (2015) 7, 074514 [arXiv:1408.5768 [hep-lat]].

[14] A. J. Lee et al. [HPQCD Collaboration], Phys. Rev. D 87 (2013) 7, 074018 [arXiv:1302.3739 [hep-lat]]. 